ISRAEL MATHEMATICAL CONFERENCE PROCEEDINGS Vol. 15, 2001

\title{
INVERTIBILITY PRESERVING LINEAR MAPS OF BANACH ALGEBRAS
}

\section{by LAWRENCE A. HARRIS}

Department of Mathematics, University of Kentucky, Lexington, Kentucky 40506

E-mail address: larry@ms.uky.edu

\begin{abstract}
This talk discusses a conjecture of R. V. Kadison and myself. Our conjecture is that each one-to-one linear map of one unital $\mathrm{C}^{*}$-algebra onto another that preserves the identity is a Jordan isomorphism if it maps the invertible elements of the first $\mathrm{C}^{*}$-algebra onto the invertible elements of the other $\mathrm{C}^{*}$-algebra. Connections are shown between this conjecture and Cartan's uniqueness theorem.
\end{abstract}

\section{Definitions and notation}

Throughout, $A$ and $B$ denote complex Banach algebras with identity (denoted by $e$ ). Put

$$
A_{\text {inv }}=\left\{x \in A: x^{-1} \text { exists }\right\}
$$

and given $x \in A$, let

$$
\sigma(x)=\left\{\lambda \in \mathbb{C}: \lambda e-x \notin A_{\text {inv }}\right\}
$$

denote the spectrum of $x$. The spectral radius of $x$ is defined by

$$
|x|_{\sigma}=\sup \{|\lambda|: \lambda \in \sigma(x)\}
$$

It is well known that $\sigma(x)$ is a non-empty compact set and that

$$
|x|_{\sigma}=\lim _{n \rightarrow \infty}\left\|x^{n}\right\|^{1 / n} \leq\|x\|
$$

Indeed, these facts are proved by applying holomorphic properties of the resolvent [12, p. 125].

By definition, $A$ is semisimple if the only element $z \in A$ satisfying $\sigma(z x)=\{0\}$ for all $x \in A$ is $z=0$. For example [12, Th. 24.8.7], if $A$ is the Banach algebra $\mathcal{L}(X)$ of all bounded linear operators on a Banach space $X$, then $A$ is semisimple. Recall that a $C^{*}$-algebra is a closed complex subalgebra $\mathfrak{A}$ of $\mathcal{L}(H)$ for some Hilbert space $H$ such that $\mathfrak{A}$ contains the adjoints of each of its elements. Throughout, capital

1991 Mathematics Subject Classification. Primary 46L05.

The Author wishes to express his gratitude to the organizers of the International Conference on Complex Analysis and Dynamical Systems, Karmiel, Israel, for their kindness and hospitality during the conference. 
German letters denote $\mathrm{C}^{*}$-algebras and all $\mathrm{C}^{*}$-algebras are assumed to contain the identity operator $I$ on $H$. Clearly, $\mathfrak{A}$ is a semisimple Banach algebra since $\sigma\left(X X^{*}\right)=0$ implies $X=0$ for $X \in \mathfrak{A}$.

If $A$ is commutative, it can be shown that $|x y|_{\sigma} \leq|x|_{\sigma}|y|_{\sigma}$ for all $x, y \in A$. Thus $A$ is semisimple if and only if the only $x \in A$ with $|x|_{\sigma}=0$ is $x=0$. For example, any closed complex subalgebra of the Banach algebra $C(S)$ of all continuous complex-valued functions on a compact Hausdorff space $S$ is semisimple since $\sigma(x)=\{x(s): s \in S\}$ for all $x \in A$. According to the Gelfand theory, every commutative semisimple Banach algebra $A$ is isomorphic to a subalgebra of $C(S)$, where $S$ is the compact set of multiplicative linear functionals on $A$ (excluding the zero functional) with the $\mathrm{w}^{*}$-topology. In particular, the multiplicative linear functionals on $A$ separate the points of $A$.

\section{Previous results and a conjecture}

The following result, obtained independently by Gleason [6] and Kahane-Żelazko $[\mathbf{1 5}, \mathbf{2 2}]$, is the first main result in this area. The proof given below is based on a special case of the Hadamard factorization theorem. A different proof has been given by Roitman and Sternfeld (see [2, p. 69-70]).

Theorem 1. Let $\phi: A \rightarrow B$ be a linear mapping with $B$ commutative and semisimple. Suppose $\phi\left(A_{\mathrm{inv}}\right) \subseteq B_{\mathrm{inv}}$ and $\phi(e)=e$. Then $\phi$ is continuous and multiplicative, i.e., $\phi(x y)=\phi(x) \phi(y)$ for all $x, y \in A$.

Proof. To see that $\phi$ is continuous, we first show that $\ell$ and $\ell \circ \phi$ are continuous whenever $\ell$ is a multiplicative linear functional on $B$ different from the zero functional. Let $\psi$ be one of these and note that $\psi\left(A_{\text {inv }}\right)$ does not contain 0 . Hence if $x \in A$ with $\|x\|=1$ and if $|\lambda|<1$, then $1-\lambda \psi(x) \neq 0$ since $e-\lambda x \in A_{\text {inv }}$ and $\ell(e)=1$. It follows that $|\psi(x)| \leq 1$ and therefore $\|\psi\| \leq 1$.

Now we use the closed graph theorem to deduce the continuity of $\phi$. Suppose $\left\{x_{n}\right\}$ is a sequence in $A$ such that $x_{n} \rightarrow x_{0}$ and $\phi\left(x_{n}\right) \rightarrow y_{0}$, where $x_{0} \in A$ and $y_{0} \in B$. Then

$$
\begin{aligned}
(\ell \circ \phi)\left(x_{n}\right) & \rightarrow(\ell \circ \phi)\left(x_{0}\right), \\
\ell\left(\phi\left(x_{n}\right)\right) & \rightarrow \ell\left(y_{0}\right),
\end{aligned}
$$

so $\ell\left(\phi\left(x_{0}\right)\right)=\ell\left(y_{0}\right)$. Since the multiplicative linear functionals on $B$ separate the points of $B$, it follows that $\phi\left(x_{0}\right)=y_{0}$, proving the continuity of $\phi$.

To establish the multiplicativity of $\phi$, it suffices to consider only the case where $B=\mathbb{C}$ since the multiplicative linear functionals on $B$ separate its points. Thus, given $x \in A$, define $f(\lambda)=\phi(\exp (\lambda x))$. Then $f: \mathbb{C} \rightarrow \mathbb{C}$ is an entire function having no zeros since every value of the exponential function on a Banach algebra is invertible. Hence there exists an entire function $g: \mathbb{C} \rightarrow \mathbb{C}$ with $f(\lambda)=e^{g(\lambda)}$ for all $\lambda \in \mathbb{C}$. Moreover, $g(0)=0$ and $\operatorname{Re} g(\lambda) \leq|\lambda|\|x\|$ for all $\lambda \in \mathbb{C}$ and it follows from a Schwarz lemma argument that $g(\lambda)=\alpha \lambda$ for some complex constant $\alpha$. 
(See [19, Lemma 10.8].) Thus,

$$
\phi\left(e+\lambda x+\frac{\lambda^{2} x^{2}}{2 !}+\cdots\right)=1+\alpha \lambda+\frac{\alpha^{2} \lambda^{2}}{2 !}+\cdots
$$

for all $\lambda \in \mathbb{C}$. Comparing coefficients, we see that $\phi(x)=\alpha$ and $\phi\left(x^{2}\right)=\alpha^{2}$, so $\phi\left(x^{2}\right)=\phi(x)^{2}$.

A slightly tricky algebraic computation shows that $\phi$ is multiplicative. Define $[x, y]=x y-y x$ and $x \circ y=x y+y x$. Since $\phi$ is a Jordan homomorphism (see below), for any $x, y \in A$,

$$
\begin{gathered}
\phi(x \circ y)=\phi(x) \circ \phi(y), \\
\phi([x, y])^{2}=\phi\left([x, y]^{2}\right)=[\phi(x), \phi(y)]^{2}=0 .
\end{gathered}
$$

Hence $\phi([x, y])=0$ so

$$
2 \phi(x y)=\phi([x, y]+x \circ y)=2 \phi(x) \phi(y) .
$$

Remark. Theorem 1 is not true if $A$ and $B$ are allowed to be real Banach algebras. For example, let $A$ be the real Banach algebra of all continuous realvalued functions $f$ on the interval $[0,1]$ with the max norm, let $B$ be the real line and define

$$
\phi(f)=\int_{0}^{1} f(t) d t
$$

Clearly $A_{\text {inv }}$ is the set of all nonzero functions in $A$ so $\phi$ satisfies the hypotheses by the intermediate value theorem. However, $\phi$ is not multiplicative.

In 1970, Kaplansky [16] posed the problem of extending Theorem 1. A natural extension to consider is where $B$ is not necessarily commutative.

A basic observation is that if $\phi: A \rightarrow B$ is a linear mapping with $\phi\left(A_{\text {inv }}\right) \subseteq$ $B_{\text {inv }}$ and $\phi(e)=e$, then $\sigma(\phi(x)) \subseteq \sigma(x)$ for all $x \in A$. Indeed, if $x \in A$ then $\phi(\lambda e-x)=\lambda e-\phi(x)$ so $\lambda \notin \sigma(\phi(x))$ whenever $\lambda \notin \sigma(x)$. If $\phi$ is also one-to-one and satisfies $\phi\left(A_{\text {inv }}\right)=B_{\text {inv }}$, then $\sigma(\phi(x))=\sigma(x)$ for all $x \in A$. Since such a $\phi$ must be surjective, one can show this by applying the above to $\phi^{-1}$.

Note that if both $A$ and $B$ are taken to be the space $M_{n}$ of all $n \times n$ matrices of complex numbers, the transpose mapping $\phi(Z)=Z^{t}$ takes the identity to the identity and invertible matrices to invertible matrices but it is not multiplicative since it reverses products. It is a classical fact that the identity and the transpose are up to similarity all the linear mappings taking the identity to the identity and invertible matrices to invertible matrices.

Theorem 2. (Marcus-Purves [17]) Let $\phi: M_{n} \rightarrow M_{n}$ be a linear mapping such that $\phi\left(G L_{n}\right) \subseteq G L_{n}$ and $\phi(I)=I$, where $G L_{n}$ is the group of invertible elements of $M_{n}$. Then there exists a matrix $A \in G L_{n}$ such that either $\phi(Z)=A Z A^{-1}$ for all $Z \in M_{n}$ or $\phi(Z)=A Z^{t} A^{-1}$ for all $Z \in M_{n}$.

This theorem was extended to the full algebra of operators on a Banach space 37 years later under the additional assumption that $\phi$ is a bijection. 
Theorem 3. (Jafarian-Sourour $[\mathbf{1 4}, \mathbf{2 1}]$ ) Let $X$ and $Y$ be complex Banach spaces and let $\phi: \mathcal{L}(X) \rightarrow \mathcal{L}(Y)$ be a linear bijection such that $\phi\left(\mathcal{L}(X)_{\mathrm{inv}}\right) \subseteq \mathcal{L}(Y)_{\mathrm{inv}}$ and $\phi(I)=I$. Then either there exists an invertible $A \in \mathcal{L}(X, Y)$ such that $\phi(Z)=$ $A Z A^{-1}$ for all $Z \in \mathcal{L}(X)$ or there exists an invertible $B \in \mathcal{L}\left(X^{*}, Y\right)$ such that $\phi(Z)=B Z^{*} B^{-1}$ for all $Z \in \mathcal{L}(X)$.

In general, a Banach algebra $A$ might be a direct sum of spaces $\mathcal{L}(X)$ on which $\phi$ is sometimes product preserving and other times product reversing. However, in all cases,

$$
\phi\left(z^{2}\right)=\phi(z)^{2}
$$

for all $z \in A$. A linear mapping $\phi: A \rightarrow B$ satisfying $\phi(e)=e$ and equation (1) is called a Jordan homomorphism and this is the conclusion sought by Kaplansky. It is easy to show [13] that if $\phi$ is a Jordan homomorphism then

$$
\begin{aligned}
\phi(z w+w z) & =\phi(z) \phi(w)+\phi(w) \phi(z) \\
\phi\left(z^{n}\right) & =\phi(z)^{n} \\
\phi(z w z) & =\phi(z) \phi(w) \phi(z) \\
\phi\left(z^{-1}\right) & =\phi(z)^{-1} \text { if } z^{-1} \text { exists }
\end{aligned}
$$

for all $z, w \in A$ and positive integers $n$. Moreover, if $\phi: A \rightarrow B$ is surjective, then $\phi$ maps the center of $A$ onto the center of $B$ provided that the center of $B$ has no nonzero nilpotents (which is true for $\mathrm{C}^{*}$-algebras).

Example 1. (Russo [20]) This example shows that an "onto" condition is needed when $A \neq B$. Define a one-to-one linear mapping $\phi: M_{2} \rightarrow M_{4}$ by

$$
\phi(Z)=\left[\begin{array}{cc}
Z & Z-Z^{t} \\
0 & Z
\end{array}\right] .
$$

Then $\phi\left(G L_{2}\right) \subseteq G L_{4}, \phi(I)=I$ and even $\sigma(\phi(Z))=\sigma(Z)$ but

$$
\phi(Z)^{2}-\phi(Z)^{2}=\left[\begin{array}{cc}
0 & \left(Z-Z^{t}\right)^{2} \\
0 & 0
\end{array}\right] \not \equiv 0
$$

Example 2. (Aupetit [1]) This example shows that semisimplicity cannot be dropped even when $\phi$ is a bijection. Let

$$
\mathfrak{A}=\left\{\left[\begin{array}{cc}
W & X \\
0 & Y
\end{array}\right]: W, X, Y \in M_{2}\right\}
$$

and define

$$
\phi(Z)=\left[\begin{array}{cc}
W & X \\
0 & Y^{t}
\end{array}\right], \text { where } Z=\left[\begin{array}{cc}
W & X \\
0 & Y
\end{array}\right]
$$

Note that $Z$ is invertible if and only if both $W$ and $Y$ are invertible. Thus $\phi: \mathfrak{A} \rightarrow \mathfrak{A}$ is a linear bijection satisfying $\phi\left(\mathfrak{A}_{\text {inv }}\right)=\mathfrak{A}_{\text {inv }}$ and $\phi(I)=I$ but

$$
\phi\left(Z^{2}\right)-\phi(Z)^{2}=\left[\begin{array}{cc}
0 & X\left(Y-Y^{t}\right) \\
0 & 0
\end{array}\right] \not \equiv 0
$$


Conjecture 1. (Harris-Kadison $[\mathbf{1 1}]$ ) Let $\mathfrak{A}$ and $\mathfrak{B}$ be $\mathrm{C}^{*}$-algebras with identity $I$ and let $\phi: \mathfrak{A} \rightarrow \mathfrak{B}$ be a linear bijection such that $\phi\left(\mathfrak{A}_{\text {inv }}\right)=\mathfrak{B}_{\text {inv }}$ and $\phi(I)=I$. Then $\phi$ is a Jordan isomorphism of $\mathfrak{A}$ onto $\mathfrak{B}$.

Theorem 1 shows that the conjecture holds when $\mathfrak{B}$ is commutative. By [4] the conjecture holds when $\mathfrak{B}$ is finite dimensional, when $\mathfrak{B}=\mathcal{L}(H)$, where $H$ is a Hilbert space, and when $\mathfrak{B}$ is the $\mathrm{C}^{*}$-algebra of compact operators on $H$ with the identity operator adjoined. Aupetit has further shown in $[\mathbf{3}]$ that the conjecture holds when $\mathfrak{A}$ and $\mathfrak{B}$ are any von Neumann algebras. Note that if the conjecture is true then by our previous comments, $\phi$ maps the center of $\mathfrak{A}$ onto the center of $\mathfrak{B}$. This is indeed the case, as shown in [11, Th. 3.6].

There is no known counterexample to the conjecture even when $\mathfrak{A}$ and $\mathfrak{B}$ are arbitrary semisimple Banach algebras.

The following result implies the automatic continuity theorem of B. E. Johnson and shows that $\phi$ is continuous in almost all the cases we consider.

Theorem 4. ([2, Theorem 5.5.2]) Let $B$ be semisimple and let $\phi: A \rightarrow B$ be a surjective linear mapping with $\phi\left(A_{\mathrm{inv}}\right) \subseteq B_{\mathrm{inv}}$ and $\phi(e)=e$. Then $\phi$ is continuous.

\section{Holomorphic methods}

In this section we use the theorem below to deduce theorems related to the conjecture. This theorem has also been used in $[\mathbf{7}, \mathbf{8}]$ to prove Kadison's characterization of isometries of $\mathrm{C}^{*}$-algebras and extensions.

Theorem 5. (Cartan's Uniqueness Theorem $[\mathbf{7}, 9]$ ) Let $X$ be a complex normed linear space and let $\mathcal{D}$ be a bounded domain in $X$. Suppose that $h: \mathcal{D} \rightarrow \mathcal{D}$ is a holomorphic function such that $h\left(x_{0}\right)=x_{0}$ and $D h\left(x_{0}\right)=I$ for some $x_{0} \in \mathcal{D}$. Then $h=I$.

By definition, $h$ is holomorphic in $\mathcal{D}$ if for each $x \in \mathcal{D}$ the Fréchet derivative $D h(x)$ exists and is a complex linear map. The theorem has been extended in [9] to hyperbolic domains but it does not hold for all unbounded domains.

ExAmple 3. This example shows that Cartan's Uniqueness Theorem does not hold when $\mathcal{D}=G L_{2}$ even when $h$ is biholomorphic and homogeneous of degree 1 . Let $\mathfrak{A}=M_{2}$ and define a biholomorphic mapping $h: \mathfrak{A}_{\mathrm{inv}} \rightarrow \mathfrak{A}_{\mathrm{inv}}$ by

$$
h(Z)=A(Z)^{-1} Z A(Z)
$$

where

$$
A(Z)=\left[\begin{array}{cc}
1 & 0 \\
f(Z) & 1
\end{array}\right], f(Z)=\frac{z_{2}^{2}}{\operatorname{det}(Z)},
$$

and where $z_{2}$ is the entry in row 1 and column 2 of $Z$. Clearly, $h(I)=I$ and $D h(I)=I$ but $h \not \equiv I$. In fact, $\sigma(h(Z))=\sigma(Z)$ and $h(\lambda Z)=\lambda h(Z)$ for all $\lambda \in \mathbb{C}$ and $Z \in \mathfrak{A}$. 
EXAMPLE 4. Let $\mathfrak{A}$ be a $\mathrm{C}^{*}$-algebra and define

$$
\mathcal{D}=\{Z \in \mathfrak{A}: \operatorname{Re} Z>0\} \text { where } \operatorname{Re} Z=\frac{1}{2}\left(Z+Z^{*}\right) .
$$

By definition, $\operatorname{Re} Z>0$ if and only if inf $\operatorname{Re} W(Z)>0$, where

$$
W(Z)=\{(Z x, x):\|x\|=1\}
$$

is the numerical range of $Z$. If $Z \in \mathcal{D}$ then $Z$ is invertible since $\sigma(Z) \subseteq \overline{W(Z)}$. Then $f(Z)=Z^{-1}$ is a biholomorphic mapping of $\mathcal{D}$ since

$$
\begin{aligned}
\operatorname{Re} Z^{-1} & =\left(Z^{-1}\right)^{*}(\operatorname{Re} Z) Z^{-1}, \\
D f(Z) W & =-Z^{-1} W Z^{-1} .
\end{aligned}
$$

Define

$$
T(Z)=(I-Z)(I+Z)^{-1}
$$

It is not difficult to show that $T$ is a biholomorphic mapping of $\mathcal{D}$ onto the open unit ball of $\mathfrak{A}$. It follows that Theorem 5 holds for $\mathcal{D}$

Theorem 6. Let $\phi: A \rightarrow B$ be a continuous linear bijective mapping such that $\phi(e)=e$. Let $\mathcal{D}$ be a domain in $A$ containing e that is holomorphically equivalent to a bounded domain. Suppose that both $\mathcal{D}$ and $\phi(\mathcal{D})$ contain the inverses of each of their elements. Then $\phi$ is a Jordan isomorphism.

Proof. By the open mapping theorem, the inverse of $\phi$ is also continuous. Define

$$
h(z)=\phi^{-1}\left(\phi\left(z^{-1}\right)^{-1}\right) .
$$

Then $h: \mathcal{D} \rightarrow \mathcal{D}$ is a biholomorphic mapping with $h(e)=e$ and $D h(e)=I$. Hence $h=I$ by Theorem 5. It follows that $\phi\left(z^{-1}\right)=\phi(z)^{-1}$ whenever $z \in \mathcal{D}$. Taking the Fréchet derivative of both sides, we have

$$
\phi\left(-z^{-1} w z^{-1}\right)=-\phi(z)^{-1} \phi(w) \phi(z)^{-1}
$$

for all $z \in \mathcal{D}$ and $w \in A$. In particular, when $w=z^{2}$ we obtain (1). By the identity theorem [12, Th. 3.16.4], this equation holds for all $z \in A$.

Note that Proposition 8 of [10] follows immediately from Theorem 6 and Example 4. In view of Example 3, there seems little hope that the previous argument will establish the conjecture, as was suggested in [10, p. 3496]. However, by applying Theorem 6 to the domain of Example 4, one can easily deduce the following result when " $\subseteq$ " is replaced by "=".

Theorem 7. ([5]) Let $\phi: \mathfrak{A} \rightarrow \mathfrak{B}$ be a linear bijection. Suppose $\phi(I)=I, \phi\left(\mathfrak{A}_{\text {inv }}\right) \subseteq$ $\mathfrak{B}_{\text {inv }}$ and $\phi\left(Z^{*}\right)=\phi(Z)^{*}$ for all $Z \in \mathfrak{A}$. Then $\phi$ is a Jordan isomorphism.

Corollary 8. Let $\phi: \mathfrak{A} \rightarrow \mathfrak{B}$ be a linear bijection. Suppose $\phi(I)=I, \phi\left(\mathfrak{A}_{\mathrm{inv}}\right) \subseteq$ $\mathfrak{B}_{\mathrm{inv}}$ and $\|\phi\| \leq 1$. Then $\phi$ is a Jordan isomorphism. 
The corollary follows since if $\phi$ is a linear mapping of one $\mathrm{C}^{*}$-algebra into another satisfying $\phi(I)=I$ and $\|\phi\| \leq 1$, then $\phi$ preserves adjoints. This can be deduced from the fact that this holds for complex-valued mappings $\phi$ (states) and that the states separate points. (Alternately, see [20, Lemma 2].) A counterexample given in [5] shows that there exists a mapping $\phi$ which is not a Jordan homomorphism even though it satisfies all the hypotheses of the corollary except that its range is not a $\mathrm{C}^{*}$-algebra.

Theorem 9. Let $\phi: A \rightarrow B$ be a linear bijection with $A$ semisimple. If $\phi(e)=e$ and $\phi\left(A_{\text {inv }}\right)=B_{\text {inv }}$, then $\phi\left(z^{2}\right)=\phi(z)^{2}$ for all $z$ in the center of $A$.

Proof. Clearly $\phi$ is continuous by Theorem 4 and $\sigma(\phi(z))=\sigma(z)$ for all $z \in A$ by hypothesis and our preliminary comments. Let $h$ be defined as in (2), put

$$
\mathcal{D}=\left\{z \in A:|z|_{\sigma}<1\right\}
$$

and define $g(z)=h(e+z)-e$ for $z \in \mathcal{D}$. Then $\sigma(h(z))=\sigma(z)$ for all $z \in A_{\text {inv }}$ so $\sigma(g(z))=\sigma(z)$ for all $z \in D$.

Hence $g: \mathcal{D} \rightarrow \mathcal{D}$ is holomorphic with $g(0)=0$ and $D g(0)=I$. By a spectral version of Cartan's Uniqueness Theorem due to Ransford [18], it follows that $g(z)=$ $z$ for all $z \in \mathcal{D} \cap Z(A)$, where $Z(A)$ is the center of $A$. Then $\phi\left(z^{-1}\right)=\phi(z)^{-1}$ for all $z \in Z(A)$ with $\|z-e\|<1$. The theorem now follows as in the proof of Theorem 6 .

\section{References}

1. B. Aupetit, Une généralisation du théorème de Gleason-Kahane-Żelazko pur les algèbres de Banach, Pacific J. Math. 85 (1979), p. 11-17.

2. _ـ A primer on Spectral Theory, Springer, Berlin, 1991.

3. __ Spectrum-preserving linear mappings between Banach algebras or Jordan-Banach algebras, J. London Math. Soc. (3) 62 (2000), 917-924.

4. _ and H. du T. Mouton, Spectrum preserving linear mappings in Banach algebras, Studia Math. 109 (1994), 91-100.

5. M-D. Choi, D. Hadwin, E. Nordgren, H. Radjavi and P. Rosenthal, On positive linear maps preserving invertibility, J. Funct. Anal. 59 (1984), 462-469.

6. A. M. Gleason, A characterization of maximal ideals, J. Analyse Math. 19 (1967), 171-172.

7. L. A. Harris, Schwarz's lemma in normed linear spaces, Proc. Nat. Acad. Sci. U.S.A. 62 1969, 1014-1017.

8. __ Bounded symmetric homogeneous domains in infinite dimensional spaces, Proceedings on Infinite Dimensional Holomorphy, Springer lecture note series vol. 364, 1974, 13-40.

9. __ Schwarz-Pick systems of pseudometrics for domains in normed linear spaces, in Advances in Holomorphy, J. A. Barroso, ed., North-Holland, Amsterdam, 1979, pp. 345-406.

10. Holomorphic mappings of domains in operator spaces, Nonlinear Analysis 30 (1997), 3493-3503.

11. and R. V. Kadison, Affine mappings of invertible operators, Proc. Amer. Math. Soc. 124 (1996), 2415-2422.

12. E. Hille and R. S. Phillips, Functional Analysis and Semi-Groups, Amer. Math. Soc. Colloq. Publ., Vol. 31, AMS, Providence, 1957. 
13. N. Jacobson and C. E. Rickart, Jordan homomorphisms of rings, Trans. Amer. Math. Soc. 69 (1950), 479-502.

14. A. A. Jafarian and A. R. Sourour, Spectrum-preserving linear maps, J. Funct. Anal. 66 (1986), $255-261$.

15. J. P. Kahane and W. Żelazko, A characterization of maximal ideals in commutative Banach algebras, Studia Math. 29 (1968), 339-343.

16. I. Kaplansky, Algebraic and analytic aspects of operator algebras, CBMS Regional Conference Series, No. 1, Amer. Math. Soc., Providence, R.I., 1970.

17. M. Marcus and R. Purves, Linear transformations on algebras of matrices: The invariance of the elementary symmetric functions, Canadian J. Math. 11 (1959), 383-396.

18. T. J. Ransford, A Cartan theorem for Banach algebras, Proc. Amer. Math. Soc. 124 (1996), $243-247$.

19. W. Rudin, Functional Analysis, McGraw-Hill, New York, 1973.

20. B. Russo, Linear mappings of operator algebras, Proc. Amer. Math. Soc.17 (1966), 1019-1022.

21. A. R. Sourour, Invertibility preserving linear maps on $\mathcal{L}(X)$, Trans. Amer. Math. Soc. 348 (1996), 13-30.

22. W. Żelazko, A characterization of multiplicative linear functionals in complex Banach Spaces, Studia Math. 30 (1968), 83-85. 